\title{
AVALIAÇÃO ANALÍTICA DE ANALISADOR DE COMBUSTÍVEL COMERCIAL NO CONTROLE DE QUALIDADE DE ETANOL E GASOLINA COMUM
}

\author{
Sthefany dos S. Sena ${ }^{a}$ e Ernesto C. Ferreira ${ }^{a *,(1)}$ \\ ${ }^{a}$ Coordenadoria do curso de Química Industrial, Instituto Federal do Espírito Santo, campus Vila Velha, 29106-010 Vila Velha - \\ ES, Brasil
}

Recebido em 10/06/2021; aceito em 13/08/2021; publicado na web em 03/09/2021

\begin{abstract}
ANALYTICAL EVALUATION OF COMMERCIAL FUEL ANALYZER IN GASOLINE AND ETHANOL FUEL QUALITY CONTROL. Brazilian news media is full of examples about fuels out of the compliances and needs to control fuel quality. This work proposed an analytical validation of a commercial near infrared photometer (Xerloq, Tech Chrom Analytical Instruments, Brazil) able to identify percentage of ethanol in fuels comparing with NBR standards. Four procedures were selected: analysis of ethanol percentage in gasoline type C samples comparing with standard method ABNT 13992; analysis of ethanol percentage in fuel ethanol samples comparing with standard method ABNT 5992; linearity correlation and selectivity of standard solution analysis. Comparisons between the photometer and standards measurements were to determine the accuracy and precision of the device which presented a little variation from the norm $\left(\mathrm{E}_{\mathrm{R}}<1.3 \%, \mathrm{~s}_{\mathrm{R}}<0.4 \%\right)$. With regard to the test for linearity, Ethanol:water mixtures was analyzed with R-squared larger than 0.999 with RMSEV less than $1 \%$. On selectivity tests, Gasoline:Ethanol:water and Methanol:Ethanol:water mixtures were measured with bias error not exceeding $5 \%$ for regulated samples. In conclusion, this fuel analyzer can be used to analyze ethanol content in Brazilian fuels with similar results comparing with classical standard volumetric and glass densimeter analysis and may indicate adulteration gasoline and ethanol samples by anomalous data.
\end{abstract}

Keywords: fuel quality; ABNT 5992; ABNT 13992; ethanol analyzer.

\section{INTRODUÇÃO}

A qualidade dos combustíveis no Brasil é um dos assuntos comentados pela mídia devido a mudanças constantes no preço de mercado internacional e no valor do dólar americano, que coincide também com o aumento das adulterações a fim de se lucrar mesmo em crises econômicas e pandêmicas. A Agência Nacional de Petróleo, Gás Natural e Biocombustíveis, ANP, é a instituição responsável por "proteger os interesses dos consumidores quanto a preço, qualidade $\mathrm{e}$ oferta de produtos, bem como especificar a qualidade dos derivados de petróleo, gás natural e seus derivados e dos biocombustíveis", ${ }^{1}$ e mantém programas de controle de qualidade de combustíveis líquidos e lubrificantes atuantes em todo território nacional e regulamenta as características destes produtos comercializados no Brasil. Atualmente, as especificações do Etanol comercializado são regulamentadas na Resolução ANP Nº 19, de maio de 2015, parte apresentadas na Tabela 1, que define três tipos de Etanol: Anidro Combustível, EAC, Hidratado Combustível, EHC, e Hidratado Combustível Premium, EHCP2. ${ }^{1}$

Segundo o painel dinâmico do Programa de Monitoramento de Qualidade dos Combustíveis, PMQC, ${ }^{2}$ foram registrados, neste ano, cerca de $2 \%$ de amostras de EHC não-conforme, sendo a principal característica de não-conformidade a massa específica ou teor de etanol. Essa não-conformidade pode ter origem na adição de outros solventes, como água e metanol, ${ }^{3} \mathrm{com}$ intuito de aumentar o volume do produto comercializado.

No caso da gasolina, ${ }^{4}$ a Resolução ANP $N^{\circ} 807$, de janeiro de 2020, estabelece as características de 4 tipos de gasolinas: gasolinas comum e premium, tipos A e C, parte apresentadas na Tabela 2.

Segundo o painel dinâmico do PMQC, ${ }^{2}$ foram registrados cerca de 1 a $3 \%$ de não-conformidades em amostras de gasolina no 2020, sendo que a principal característica não-conforme é o teor de EAC. Essa não-conformidade pode ocorrer pela adição de solventes,,${ }^{5-11} \mathrm{de}$ tráfico de gasolinas importadas, ${ }^{12}$ e adição de EHC. ${ }^{10,11}$

Os metódos analíticos oficiais para análise de teor etanólico em gasolinas e EHC são normatizados pela Associação Brasileira de Normas Técnicas, ABNT, codificados como NBR13992 ${ }^{13} \mathrm{e}$ NBR5992,${ }^{14}$ respectivamente. Para análise de teor etanólico em gasolina, utiliza-se um método volumétrico, usando uma proveta com tampa calibrada em balança analítica, com extração do etanol presente com uma solução de $\mathrm{NaCl} 10 \%$, com uma frequência analítica de

Tabela 1. Especificações do etanol para comercialização ${ }^{1}$

\begin{tabular}{|c|c|c|c|c|c|}
\hline \multirow{2}{*}{ Característica } & \multirow{2}{*}{ Unidade } & \multicolumn{3}{|c|}{ Limite } & \multirow{2}{*}{$\begin{array}{c}\text { Método de Análise } \\
\text { NBR }\end{array}$} \\
\hline & & EAC & $\mathrm{EHC}$ & EHCP2 & \\
\hline Massa específica a $20^{\circ} \mathrm{C}$ & $\mathrm{kg} / \mathrm{m}^{3}$ & 791,5 máx. & 805,2 a 811,2 & 799,7 a 802,8 & $\begin{array}{c}5992 \\
15639\end{array}$ \\
\hline Teor alcoólico & $\%$ massa & 99,3 mín. & 92,5 a 94,6 & 95,5 a 96,5 & $\begin{array}{c}5992 \\
15639\end{array}$ \\
\hline Teor de etanol, mín. & $\%$ volume & 98,0 & 94,5 & 96,3 & 16041 \\
\hline Teor de água, máx. & $\%$ massa & 0,7 & 7,5 & 4,5 & $\begin{array}{l}15531 \\
15888\end{array}$ \\
\hline Teor de metanol, máx. & $\%$ volume & 0,5 & & & 16041 \\
\hline
\end{tabular}

\footnotetext{
*e-mail: ernesto.ferreira@ifes.edu.br
} 
Tabela 2. Especificações de gasolina ${ }^{4}$

\begin{tabular}{|c|c|c|c|c|c|c|}
\hline \multirow{3}{*}{ Característica } & \multirow{3}{*}{ Unidade } & \multicolumn{4}{|c|}{ Limite } & \multirow{3}{*}{$\begin{array}{c}\text { Método de } \\
\text { Análise }\end{array}$} \\
\hline & & \multicolumn{2}{|c|}{ Comum } & \multicolumn{2}{|c|}{ Premium } & \\
\hline & & A & $\mathrm{C}$ & A & $\mathrm{C}$ & \\
\hline Teor de Etanol Anidro Combustível (EAC) & $\%$ volume & $<1$ & $27 \pm 1^{*}$ & $<1$ & $25 \pm 1 *$ & 13992 \\
\hline Massa específica a $20^{\circ} \mathrm{C}$, mín. & $\mathrm{kg} / \mathrm{m}^{3}$ & 688,9 & 715,0 & 691,5 & 715,0 & $\begin{array}{c}7148 \\
14065\end{array}$ \\
\hline Teor de Enxofre, máx. & $\mathrm{mg} / \mathrm{kg}$ & - & 50 & - & 50 & - \\
\hline Teor de Metanol, máx & $\%$ volume & 0,5 & & & & 16041 \\
\hline
\end{tabular}

*MAPA Portaria $\mathrm{N}^{\circ}$ 75, de maio de 2015.

3 medidas por hora e precisão com desvio-padrão relativo entre 0,4 a $0,8 \% .{ }^{13}$ Para análise de massa específica do EHC, utiliza-se um densímetro e termômetro de vidro calibrados, com uma frequência analítica de 10 medidas por hora e precisão com desvio-padrão relativo entre 0,2 a $0,3 \% .^{14}$

O método proposto pelo fabricante do Analisador de Combustíveis apresenta uma frequência analítica de 60 medidas por hora, com o instrumento calibrado de fábrica. Esse instrumento analítico baseia-se na absorção da radiação na região do NIR (Near Infrared, Infravermelho próximo) pelas ligações $\mathrm{CO}-\mathrm{H}$ presentes na molécula de etanol.

Esse instrumento ou fotômetro NIR já foi utilizado em outro trabalho nesta revista, ${ }^{11}$ em análises de amostras de combustíveis apreendidas pela Polícia Cívil do estado do Espírito Santo. Os resultados foram comparados com métodos multivariados com um espectrofotômetro NIR e H-NMR (Hydrogen Nuclear Magnetic Resonance, Ressonância Magnética Nuclear de prótons), e nenhuma das técnicas analíticas apresentou resultados totalmente concordantes com os relatórios das análises de laboratórios associados com ANP. Os resultados do fotômetro NIR indicaram que a maioria das amostras de gasolina analisadas apresentavam valores acima do regulamentado. E, para as amostras de EHC, apenas um resultado foi considerado não-conforme, mas concordante com os resultados dos laboratórios associados com ANP. Dessa forma, uma avaliação analítica do fotômetro NIR na análise de combustíveis poderia elucidar as diferenças de resultados obtidos ${ }^{11}$ e identificar as suas limitações.

\section{PARTE EXPERIMENTAL}

\section{Amostragem e preparo de soluções}

Foram coletadas 20 amostras de Gasolina premium tipo C e 22 amostras de EHC com galões apropriados (INMETRO) em postos sorteados aleatoriamente de um banco de dados (Libre Office 4) elaborado pelo projeto "Adulteração de Combustíveis na Região Metropolitana de Vitória" com fomento do Programa Petrobrás de Formação de Recursos Humanos (Convênio Ifes-PetrobrásFACTO).${ }^{15}$ As amostras codificadas foram reservadas em frascos de vidro âmbar a $4{ }^{\circ} \mathrm{C}$.

As soluções padrão foram preparadas em balões volumétricos e em balança analítica (Shimadzu U, Japão). Os reagentes utilizados foram de grau analítico ( $\mathrm{NaCl}$ P.A., Vetec, Brasil, Etanol Absoluto, Dinâmica, Brasil), grau HPLC (Metanol, Sigma-Aldrich, EUA) e água deionizada.

\section{Ensaios e testes}

As amostras de gasolina e EHC foram analisadas segundo os procedimentos das normas NBR $13992^{13}$ e NBR 5992, ${ }^{14}$ respectivamente, e do procedimento do fabricante do Analisador de
Combustíveis, baseado em fotometria NIR (Xerloq, Tech Chrom Instrumentos Analíticos, Brasil $)^{16}$ em laboratório a $20{ }^{\circ} \mathrm{C}$. A massa específica e a temperatura das amostras de EHC foram medidas com densímetro e termômetro de vidro calibrados. As variações de massa específica com temperatura medida foram corrigidas com software "Tabela Alcoolométricas" disponibilizada pela ABNT, ${ }^{16}$ como também a conversão da massa específica medida para teor de etanol na amostra. Todas as medidas foram realizadas em triplicata por dois analistas diferentes e equipamentos diferentes.

\section{Avaliação analítica}

\section{Linearidade}

Para o estudo em etanol combustível, foram preparadas soluções padrão de etanol em água nas concentrações de 36,0 a 99,7\% (m/m). Essas soluções foram analisadas no fotômetro NIR a $20^{\circ} \mathrm{C}$.

Para estudo em gasolina tipo $\mathrm{C}$, foram preparadas soluções padrão de gasolina tipo $\mathrm{C}(27,0 \%, \mathrm{~V} / \mathrm{V})$ com adição de etanol absoluto $(99,8 \%, \mathrm{~V} / \mathrm{V})$ entre $30-79 \%$ (V/V). Essas soluções foram analisadas no fotômetro NIR a $20^{\circ} \mathrm{C}$.

\section{Seletividade}

Foram preparadas soluções de etanol (85-98\%, massa), metanol (0-5\%, massa) e água (5-15\%, massa) e analisadas no fotômetro NIR a $20{ }^{\circ} \mathrm{C}$.

\section{RESULTADOS E DISCUSSÃO}

\section{Avaliação analítica}

\section{Exatidão e precisão}

As amostras foram analisadas pelos métodos oficiais e pelo fotômetro NIR e seus resultados foram avaliados sobre a exatidão pelo erro absoluto. Os valores de erro relativo calculados ente as análises de gasolina não foram superiores a $0,1 \%$, conforme ilustra a Figura $1 \mathrm{~A}$. Na maioria das análises de EHC, os valores de erro relativo não foram superiores a $1,3 \%$, apenas uma amostra apresentou um valor anômalo ou não-conforme pelo fotômetro NIR, conforme ilustra a Figura 1B.

A precisão foi avaliada a partir de estudos de repetibilidade e reprodutibilidade usando o fotômetro NIR, sendo que os valores de desvio-padrão relativo calculados entre as análises não foram superiores a 0,2 e 0,4\%. As normas NBR 13992 e 5992 apresentam valores de repetibilidade e reprodutibilidade semelhantes, 0,4 e $0,8 \%$, e 0,2 e $0,3 \%$, respectivamente.

\section{Linearidade}

O fotômetro apresentou uma relação linear entre as medidas em etanol combustível com soluções padrão com teor de etanol entre $36-99,7 \%(\mathrm{~m} / \mathrm{m})$, conforme ilustra a Figura 2A. O coeficiente de determinação calculado foi superior a 0,999 e com raiz quadrado do 



Figura 1. Comparação dos resultados das análises de (A) gasolina e (B) EHC
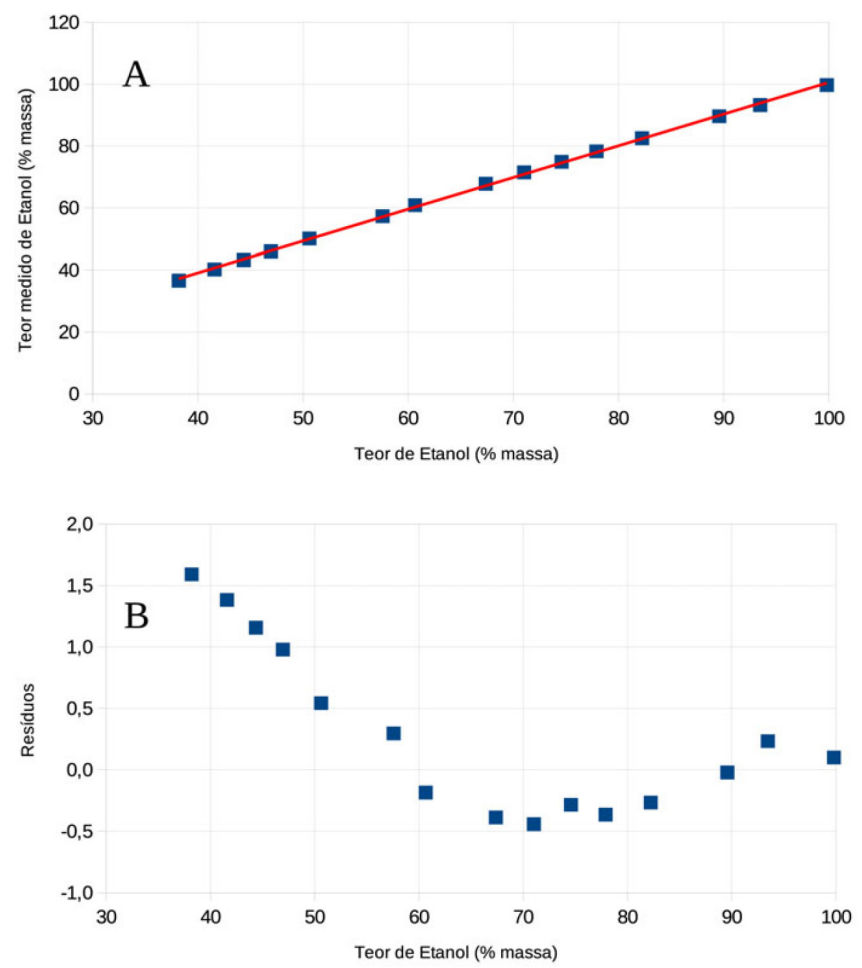

Figura 2. Estudo de linearidade com soluções padrão de etanol: (A) curva de calibração com $R^{2}=0,9994$ e (B) gráfico de resíduos com $R M S E V(\%)=0,8$

erro quadrático, RMSEV, menor que $1 \%$ entre os teores de etanol das soluções padrão e as medidas.

Avaliando-se o teor de etanol em gasolina tipo C, o fotômetro também apresentou uma relação linear entre as medidas e soluções padrão de gasolina e etanol absoluto com teor de etanol (V/V) entre $30-79 \%$, conforme ilustra a Figura 3 . O coeficiente de determinação calculado também foi superior a 0,999 com RMSEV de 2,7\% entre os teores de etanol e as medidas.


Figura 3. Estudo de linearidade com soluções padrão de gasolina tipo $C e$ etanol absoluto: (A) curva de calibração com $R^{2}=0,9994$ e (B) gráfico de resíduos com $\operatorname{RMSEV}(\%)=2,7$

\section{Seletividade}

O fotômetro foi avaliado por sua seletividade através de medidas de soluções padrão preparadas com gasolina tipo C e EHC, e soluções padrão de etanol, metanol e água. A Figura 4 apresenta diagramas ternários com coloração nos pontos representando o erro relativo das medidas de teor de etanol nos dois experimentos. Na análise do etanol em gasolina, a adição de EHC, e consequentemente, o aumento das concentrações de etanol e água nas soluções, aumenta consideravelmente o erro relativo, mesmo em quantidades pequenas, conforme ilustram a Figura 4A. Na análise de etanol em EHC, podese verificar que os erros relativos são maiores que $5 \%$ na presença de quantidades 10 vezes maiores de metanol permitido pela ANP.

Como o fotômetro realiza medidas de absorção no NIR relativa as vibrações $\mathrm{O}-\mathrm{H}$, a adição de substâncias que apresentam tal ligação química aumentará a absorção da radiação com absortividades molares diferentes do etanol. Isso explica por que o erro relativo das medidas aumenta com a adição do EHC na gasolina tipo $\mathrm{C}$, que pode conter até $7 \%$ de água, e com a adição de metanol e água no EHC.

Dessa forma, a adulteração de gasolina tipo $\mathrm{C}$ com adição de nafta petroquímica e correção do teor de etanol com EHC, deve aumentar o valor medido de EAC pelo fotômetro pela adição de água, possivelmente, registrando uma amostra não-conforme, ao contrário do resultado da análise volumétrica ABNT 13992. E a adulteração de EHC pela adição de metanol e água também resulta em uma medida maior de teor etanólico pelo fotômetro, ao contrário da análise volumétrica ABNT 5992.

Por exemplo, uma prática criminosa comum é o furto de combustíveis pelos próprios motoristas dos caminhões tanque que transportam os combustíveis do centro de distribuição da PETROBRAS para os postos de distribuição. Segundo relatos de proprietários de postos de distribuição e notícias na mídia, o motorista utiliza de bombonas de $200 \mathrm{~L}$ para retirar a gasolina tipo C do compartimento de $20.000 \mathrm{~L}$, substituindo este volume por $200 \mathrm{~L}$ EHC retirados de outro compartimento de $20.000 \mathrm{~L}$, e 

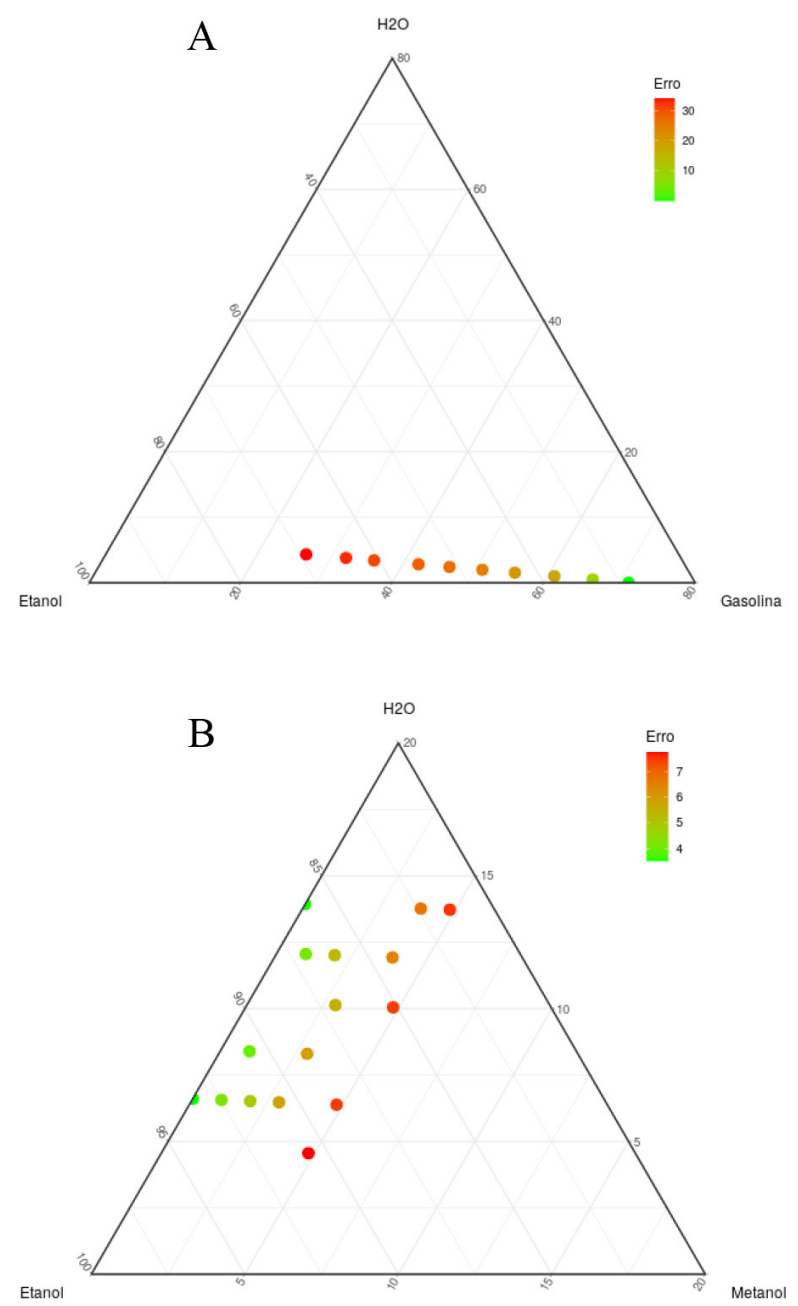

Figura 4. Estudo de seletividade do fotômetro NIR: (A) Gasolina C adulterado com EHC; (B) EHC adulterado com metanol

adicionando 200 L de água potável no compartimento de EHC. As mudanças de teores de etanol são da ordem de $0,1 \%$, variação que está dentro da precisão dos métodos oficiais. Contudo, o aumento das quantidades de água na gasolina e no EHC poderá ser indicado pela medida com o fotômetro, que acusará valores maiores de etanol nesses combustíveis. Outro exemplo, é adulteração pela adição de nafta petroquímica, solvente usado na indústria de tintas e vernizes que apresenta taxas de impostos menores que os combustíveis e com composição química semelhante à gasolina tipo A. Nesse caso, a adulteração ocorre em centrais de distribuição que comercializam combustíveis e solventes, cuja prática consiste na adição de nafta à gasolina tipo $\mathrm{C}$ e ajuste do teor de etanol com EHC. Novamente, a análise volumétrica poderá não verificar a inconsistência, ao contrário do fotômetro, que medirá também a presença da água e o teor de etanol medido será maior que o ajustado. Esse comportamento do fotômetro frente a amostras não-conformes e/ ou adulteradas poderia ser utilizado como um dos indicativos para realizar as demais análises físico-químicas para a confirmação da não-conformidade, diminuindo os custos da fiscalização.

Contudo, como já descrito anteriormente, a aplicação do fotômetro em trabalho anterior ${ }^{11}$ apresentou discrepâncias com os resultados esperados, principalmente na análise de teor etanólico em gasolina. Conforme esse trabalho, ${ }^{11}$ apenas 14 amostras se mostraram dentro da conformidade das 76 analisadas. Inclusive, amostras de gasolina tipo A apresentaram resultados de 34 e $32 \%$ de teor de etanol. Esses resultados não fazem sentido, já que essas amostras de gasolina tipo A não deveriam apresentar etanol na sua composição. Avaliando-se os espectros de NIR apresentados na Figura 3A desse artigo, ${ }^{11}$ todas as amostras analisadas apresentam absorções na região de $1300-1680 \mathrm{~nm}$, característicos do etanol e não presente em espectros NIR de gasolina importadas. ${ }^{17-19}$ Dessa forma, a possível discrepância pode estar nas amostras consideradas gasolina tipo A e não nos resultados do fotômetro avaliado, e os resultados nãoconformes apresentados pelo fotômetro podem indicar algum tipo de adulteração mascarado pela adição de EHC.

\section{CONCLUSÕES}

Conclui-se, portanto, que o Fotômetro Analisador de Combustível, Tech Chrom Instrumentos Analíticos Ltda demonstrou ser eficiente na proposta de sua criação para a realização das análises rápidas substituindo as normas ABNT 5992 e 13992, sendo utilizado, portanto, como forma preliminar de investigação de não conformidades que possam ser consideradas adulterações. Outro aspecto é relacionado à praticidade do aparelho que substitui várias vidrarias de laboratório, como provetas, densímetros, termômetros e suas respectivas calibrações, fazendo o trabalho de duas normas em apenas um aparelho.

Comparando-se com as análises normatizadas, os erros relativos não ultrapassaram 1,3\% para a avaliação da exatidão do instrumento. Comparando-se a precisão, os valores de desvio-padrão não foram superiores a $0,4 \%$. No estudo de linearidade, as resposta foram lineares na faixa de $36-99,7 \%(\mathrm{~m} / \mathrm{m})$ para o teor de etanol em EHC $\left(\mathrm{R}^{2}=0,9994, \mathrm{RMSEV}=0,8 \%\right.$ ) e na faixa de $29-78 \%$ (V/V) em gasolina tipo $\mathrm{C}\left(\mathrm{R}^{2}=0,9994\right.$, $\left.\mathrm{RMSEV}=2,7 \%\right)$. No estudo de seletividade, o instrumento teve um aumento expressivo no erro relativo com a adição de EHC em gasolina tipo C e metanol e água em EHC. Esse comportamento é interessante na fiscalização, pois pode indicar algum tipo de adulteração pela adição de solventes, água e metanol.

\section{AGRADECIMENTOS}

Os autores agradecem ao Programa Petrobrás de Formação de Recursos Humanos (Convênio Ifes-PB-Facto 2014-2016) e ao CNPq (488.341/2013-2) pelo suporte financeiro.

\section{REFERÊNCIAS}

1. Agência Nacional de Petróleo, Gás Natural e Biocombustíveis. Resolução ANP No 19, DE 15.4.2015 - DOU 16.4.2015. Disponível em https://atosoficiais.com.br/anp/resolucao-n-192015 ?origin $=$ instituicao $\& q=$ RANP $\% 2019 \% 202015$, acessado em Outubro de 2020.

2. Agência Nacional de Petróleo, Gás Natural e Biocombustíveis. Painel Dinâmico PMQC. Disponível em http://www.anp.gov.br/qualidadeprodutos/158-programas-de-monitoramento/5977-painel-dinamico-dopmqc, acessado em Outubro de 2020.

3. Martins, G. B.; Montenegro, M. A.; Suarez, P. A. Z. Quim. Nova 2014, 38,280 .

4. Agência Nacional de Petróleo, Gás Natural e Biocombustíveis. RESOLUÇÃO ANP No 807, DE 23.01.2020 - DOU 24.01.2020 RETIFICADA DOU 27.01.2020. Disponível em https://atosoficiais.com. br/anp/resolucao-n-807-2020-estabelece-a-especificacao-da-gasolinade-uso-automotivo-e-as-obrigacoes-quanto-ao-controle-da-qualidadea-serem-atendidas-pelos-agentes-economicos-que-comercializarem-oproduto-em-todo-o-territorio-nacional?origin=instituicao\&q=Resolu \% C3\%A7\%C3\%A3o\%20807\%202020, acessada em Outubro de 2020.

5. Takeshita, E. V.; Rezende, R. V. de P.; Souza, S. G.; Souza, A. A. U. de. Fuel 2008, 87, 2168. 
6. Pedroso, M. P.; de Godoy, L. A. F.; Ferreira, E. C.; Poppi, R. J.; Augusto, F. J. Chromatogr. A 2008, 1201, 176.

7. Godoy, L. A. F. de; Ferreira, E. C.; Pedroso, M. P.; Fidélis, C. H. de V.; Augusto, F.; Poppi, R. J. Analytical Letters 2008, 41, 1603.

8. Vempatapu, B. P.; Kanaujia, P. K. Trends in Analytical Chemistry 2017, $92,1$.

9. Wiedemann, L. S. M.; d'Ávila, L. A.; Azevedo, D. A. Fuel 2005, 84, 467.

10. Mendes, G.; Barbeira, P. J. S. Fuel 2013, 112, 163.

11. Tosato, F.; Barros, E. V.; Cunha, D. A.; Santos, F. D.; Corrêa, T.; Nunes, A.; Jastrow, I.; Silverol, M. A.; Pinheiro, L. U.; Seabra, A. C.; Filgueiras, P. R.; Neto, A. C.; Wang, L.; Ferreira, E. C.; Romão, W. Quim. Nova 2020, $43,155$.
12. Godoy, L. A. F. de; Pedroso, M. P.; Hantao, L. W.; Augusto, F.; Poppi, R. J. J. Braz. Chem. Soc. 2013, 24, 645.

13. https://www.abntcatalogo.com.br/norma.aspx?ID=345026, acessado em Outubro de 2020

14. https://www.abntcatalogo.com.br/norma.aspx?ID=364212, acessado em Outubro de 2020.

15. https://ifes.edu.br/noticias/146-aluno/14233-programa-petrobras-deformacao-de-recursos-humanos, acessada em Outubro de 2020.

16. http://xerloq.com.br/, acessado em Outubro de 2020.

17. Balabin, R. M; Safieza, R. Z.; Lomakina, E. I. Chemometrics and Intelligent Laboratory Systems 2007, 88, 183.

18. Chung, H.; Lee, H.; Jun, C. Bull. Korean Chem. Soc. 2001, $22,37$.

19. Bohács, G.; Ovádi, Z.; Salgó, A. J. Near Infrared Spectrosc. 1998, 6, 341. 